Célia Landmann Szwarcwald 1

Francisco Inácio Bastos 1

Carla Lourenço Tavares de Andrade 1

\section{Medidas de desigualdad en salud: la discusión de algunos aspectos metodológicos con una aplicación para la mortalidad neonatal en el Municipio de Rio de Janeiro, 2000}

\author{
Health inequality indicators: a discussion \\ of some methodological approaches as applied \\ to neonatal mortality in the Municipality \\ of Rio de J aneiro, 2000
}

1 Departamento de Informação em Saúde, Centro de Informação Científica e Tecnológica, Fundação Oswaldo Cruz. Av. Brasil 4365, Rio de Janeiro, RJ 21045-900, Brasil. celials@fiocruz.br
Abstract Epidemiology has investigated the relationship between health status and different social and economic factors ever since the fiel d emerged. Studies have consistently shown that the populati on's health status bears a strong social gradient, invariably unfavorable to the less privileged groups. Increasing interest in understanding and characterizing health inequalities has broadened the discussion in the recent literature on appropriate concepts and methodological procedures for measuring differences in health status according to socioeconomic level. This study presents a critical assessment of health inequality indicators, focusing on the following: the redistribution principle and its application to health status; the influence of income inequality; epidemiological and statistical approaches to the problem; and evaluation of health system performance in reducing health inequalities. As an example, inequalities in the neonatal mortality rate are analyzed in the city of Rio de Janei ro, Brazil, 2000, according to the mother's level of schooling, reviewing the minimum requisites for defining an adequate health inequality indicator.

Key words Equity; Measures; Health Conditions; Infant Mortality

Resumen La relación entrelas condiciones de salud de la población y los diferentes factores sociales y económi cos está siendo investi gada por la Epidemi ología desde sus comi enzos. De modo consensual, los estudios están evidenciando quela salud de la población presenta un fuerte gradiente social, invariablemente desfavorable a los grupos social mente menos privilegiados. El interés creciente en entender y caracterizar las desi gual dades en salud ha ampliado la discusión, en la literatura reciente, sobre la conceptualización y la metodología propias para medir las diferencias de las condiciones de salud por cada grupo socioeconómico. En el presente trabajo, se realiza una apreciación crítica de la evaluación de desigualdades soci oeconómicas en salud, teniéndose en consideración los siguientes aspectos: el principio de redistribución; la influencia de la distribución derenta; el análisis del problema bajo la óptica de los modelos epidemiológicos y estadísticos; Ia actuación del sistema de salud en la reducción de las desigualdades. Como ejemplo, son analizadas las desi gualdades dela mortalidad neonatal en el Municipio de Río deJaneiro, 2000, según el grado de educación de la madre. Los índices son comparados, examinándose los atri butos míni mos necesarios con la defini ción de las medidas adecuadas a las desi gualdades socioeconómi cas en salud.

Palabras clave Equidad; Medidas; Condiciones de Salud; Mortalidad Infantil 
Introducción

La relación entre las desigualdades de salud de la población y los factores sociales y económicos está siendo investigada por la Epidemiología desde sus orígenes (Berkman \& Kawachi, 2000). De manera general, las evidencias apuntan a un fuerte gradiente social en el ámbito de la salud de la población, invariablemente desfavorable para los grupos social mente menos privilegiados (Adler et al., 1993; Duncan, 1996; Evans et al., 1994; Kaplan, 1996).

El estudio específico de las desigualdades en salud adquirió mayor énfasis a partir de la publicación del "Relatório Black", cuando Townsend \& Davidson (1982) evidenciaron grandes disparidades sociales en la situación de salud de la población británica. Desde entonces, diversos investigadores se han dedicado a analizar las diferencias en las condiciones de salud o en el acceso a los servicios de salud de acuerdo con la división de la población por nivel socioeconómico (NSE), sea este medido por renta, educación, ocupación o posición en la jerarquía social (Chandola, 2000; Kaplan \& Keil, 1993; Knust et al., 1995; Koskinen, 1985, apud Mackenbach \& Kunst, 1997; LeGrand \& Rabin, 1986, apud Mackenbach \& Kunst, 1997; Mackenbach et al., 1997; Pamuck, 1985; Wagstaff, 2000).

Ante la evidencia consistente de que personas desfavorecidas socialmente tienen peor situación de salud, al rededor del mundo y persistente en el tiempo, la atención a las desigualdades han sido tema de discusión en la agenda política de la Organización Mundial de la Salud (OMS) (Acheson, 2000; Gwatkin, 2000). Teniendo como objetivo reducir las diferencias en las condiciones de salud entre los países y entre los subgrupos poblacionales dentro de los países, a través de la mejora del nivel de salud de las naciones y grupos menos favorecidos, la OMS propuso, recientemente, la inclusión de la medición de las desigualdades en salud como una de las dimensiones de la evaluación del desempeño de los sistemas de salud (WHO, 2000).

El principal argumento para la reducción de las desigualdades de la salud se basa en el principio de equidad, que incorpora la dimensión de justicia social. Conforme fue concebido por Whitehead (1992:433): "equidad en saIud implica que idealmentetodos deben tener una justa oportunidad de alcanzar todo su potencial de salud y nadie debeestar en desventaja para alcanzar este potencial, si esto pudiera ser evitado".

Como argumentación secundaria, se sugiere que la reducción de las desigualdades de salud en una sociedad trae beneficios que no se restringen a los subgrupos poblacionales más desfavorecidos, contribuyendo también substancial mente para elevar los niveles medios del estado de salud de la población como un todo (Mackenbach \& Kunst, 1997). En el mismo contexto, Woodward \& Kawachi (2000) aportan la idea de que las marcadas disparidades en salud en una sociedad son perjudiciales a todos sus miembros, no restringiéndose a los límites de los segmentos menos privilegiados.

Cabe observar que, si por un lado, la meta de reducción de las desigualdades en salud está apoyada en los fundamentos de justicia social, por otro, las teorías de justicia no se traducen, necesariamente, en la práctica, en acciones concretas de reducción de estas desigualdades (Woodward \& Kawachi, 2000). En este sentido, el interés creciente en entender y caracterizar las desigualdades en salud ha ampliado la discusión, en la literatura reciente, sobre la conceptualización y la metodología propias para evaluar y dimensionar las diferencias de la situación de salud en una sociedad, así como las etapas indispensables a la implementación de acciones basadas en evidencias empíricas y susceptibles de evaluación.

En el presente trabajo, se objetiva realizar una apreciación crítica de las medidas de desigualdad en salud, teniéndose en consideración los siguientes aspectos: el principio de la redistribución y su aplicación a la salud; los determinantes socioeconómicos de las desigualdades en salud y la influencia de la distribución de renta; el tratamiento del problema bajo la óptica de los modelos epidemiológicos y estatísticos; y los atributos mínimos necesarios a la definición de medidas de desigualdad en salud. Al final del texto, a título de ejemplificación, las propiedades de las medidas de desigualdad en salud son discutidas por medio del análisis de resultados de estudio sobre la mortalidad infantil precoz, en el Municipio de Río de Janeiro, en el año 2000.

\section{Desigualdades socioeconómicas de la salud: concepto}

En gran parte de los trabajos contemporáneos de revisión sobre el tema, las desigualdades en salud son consideradas como las diferencias en las condiciones de salud evidenciadas en distintos subgrupos poblacionales. Si bien que los subgrupos poblacionales puedan ser definidos y constituidos a partir de varias dimensiones como la biológica, la social, la económica, o la étnica, generalmente, se procede a los análisis a partir de una dimensión "socioeconómica" (Mac- 
kenbach \& Kunst, 1997; Wagstaff et al., 1991), utilizándose, con relativa frecuencia, el término "desigual dades socioeconómicas de la salud".

En la vertiente complementaria, se sitúan los estudios de apreciación de las desigualdades geográficas de salud. A pesar de que la división geográfica no pueda ser utilizada como proxy para la división socioeconómica, existe, por norma general, una fuerte asociación entre el padrón de las condiciones de vida y el padrón geográfico de ocupación del espacio (Santos, 1994), influenciando, conjuntamente, la situación de salud. Por otro lado, la introducción de las técnicas de geoprocesamiento y de análisis estadístico espacial ha facilitado la detección de áreas donde existe mayor riesgo de muerte y de enfermedades, permitiendo evidenciar con mayor nitidez las desigualdades espaciales de salud.

Bajo la óptica estadística, el término “desigualdades" es utilizado para indicar diferencias sistemáticas entre los miembros de una determinada población o entre subgrupos poblacionales, pudiendo el término ser utilizado en el sentido puramente cuantitativo. Desde el punto de vista de la inferencia estadística, la hipótesis nula a ser considerada es la de homogeneidad de la probabilidad de incidencia de un determinado suceso (relacionado con la salud) en la población condicionada a las características del individuo o del grupo al cual él pertenece (Manor et al., 1997).

De manera distinta, algunos autores proponen que las desigualdades de la salud deben ser evaluadas mediante índices compuestos de las variaciones del estado de salud (medido, por ejemplo, por la esperanza de vida al nacer o por la esperanza de vida saludable) entre individuos en la población, sin tener en consideración, a priori, las características del individuo, esto es, los posibles determinantes de las diferencias individuales en el estado de salud (Murria et al., 1999). Entretanto, la utilización de tales índices está siendo severamente criticada en la literatura, ya que tales índices son directa y fuertemente influenciados por las diferencias socioeconómicas existentes en la población (Wagstaff et al., 1991).

El índice propuesto por la OMS para el análisis de las desigualdades de Salud se basa en la medición de las variaciones interindividuales del nivel de salud (Gakidou et al., 2000) y ha sido criticado por no tener en consideración los determinantes de las desigualdades, siendo antes sensible a las desigualdades en el ámbito de la distribución de renta que propiamente al desempeño del sistema de salud (Braveman et al., 2001; Howelling et al., 2001; Szwarcwald, 2002).
La proposición de la OMS levanta, sin embargo, un punto fundamental en el ámbito de la problemática de la medición de las desigualdades en salud. Si, por un lado, es un hecho que la dimensión socioeconómica necesita ser tenida en consideración, por otro, la actuación del sistema de salud, traducida en las acciones de salud, es también esencial para la ponderación de la cuestión.

Medidas de desigualdad en salud: una revisión sinóptica

Diversos métodos pueden ser utilizados en la medida de las desigualdades de las condiciones de salud de una población. Las ventajas y desventajas de las distintas estrategias metodológicas han sido discutidas en la literatura especializada reciente.

Algunas de las medidas pueden ser consideradas simples, en el sentido en que se basan, exclusivamente, en calcular razones entre tasas de mortalidad o diferencias en las tasas de mortalidad en grupos situados en puntos extremos de las sociedades (Towsend \& Davidson, 1982).

Otras medidas de desigualdad en salud integran el elenco de medidas utilizadas habitualmente por la Epidemiología para evaluar diferencias en los riesgos de muerte o de enfermedad, como la razón de los productos cruzados (odds-ratio) o el riesgo atribuible (population-attributable risk), conforme fue apuntado por Macken bach \& Kunst (1997).

Ya las medidas más sofisticadas utilizan el orden del nivel socioeconómico, representado como una variable ordinal, de modo que sea posible relacionar cuantitativamente la variación de los indicadores de salud con la variación del nivel socioeconómico, como, por ejemplo, el "coeficiente angular de la desigualdad" (slope index of inequality) $u$ otras medidas basadas en procedimentos de regresión (Pamuck, 1985).

Se dispone todavía de procedimientos alternativos, basados en medidas estadísticas utilizadas para comprobar hipótesis de homogeneidad de las probabilidades de incidencia de agravios de salud en distintos subgrupos poblacionales, definidos a partir de atributos socioeconómicos. Entre estos, el más utilizado es el "índice de disimilaridad" (dissimilarity index) (Koskinen, 1985, apud Mackenbach \& Kunst, 1997).

De forma semejante al coeficiente de Gini utilizado para medir las desigualdades en la distribución de renta -, fueron propuestos, en 
la década de los 80, índices compuestos para representar la distribución del estado de salud en la población, midiendo variaciones interindividuales del estado de salud. Con el propósito de medir desigualdades en salud, el coeficiente de Gini, fue utilizado, primeramente, por LeGrand \& Rabin (1986, apud Mackenbanch \& Kunst, 1997). Cabe observar, no obstante, que la utilización del coeficiente de Gini con tal propósito fue severamente criticada en la literatura por dos razones principales: el índice es fundamentado en el principio de "redistribución de la salud"; además, el índice, por medir variaciones entre individuos de los indicadores de salud, está fuertemente afectado por la magnitud de las desigualdades del nivel socioeconómico en la población (Wagstaff et al., 1991).

Entendiendo el concepto de "desigualdades en salud" como "desigualdades socioeconómicas de la salud", Wagstaff et al. (1991) elaboraron una revisión de posibles medidas de desigualdades en salud, considerando tres atributos absolutamente necesarios para su adecuada medición: (a) que la medida refleje la dimensión socioeconómica de las desigualdades en salud; (b) que la medida refleje las experiencias de la totalidad de la población, y no sólo la de los estratos sociales situados en las extremidades del gradiente socioeconómico; (c) que la medida sea sensible a cambios en la distribución de la población por grupo socioeconómico. De acuerdo con estos tres requisitos, los autores proponen que sean utilizados sólo dos indicadores para la medición de las desigualdades en salud: el "coeficiente angular de desigualdad" y el "índice de concentración”, cuyas definiciones están presentadas en el Tabla 1, demostrando, todavía, que existe una relación entre los dos indicadores: el primero es igual al segundo dividido por la variante de la variable ordinal que mide las diferencias en el nivel socioeconómico.

Dando continuidad al trabajo de revisión de Wagstaff et al. (1991), Mackenback \& Kunst (1997) describen una serie de medidas de desigualdades socioeconómicas de la salud, que pueden ser estimadas como medidas absolutas o relativas, y distinguen aún las "medidas de efecto" de las "medidas de impacto total", que, a diferencia de las primeras, miden el efecto global, estimado por mediación de los efectos parciales de las distintas categorías de NSE.

Adicionalmente, Mackenback \& Kunst (1997) enfatizan la importancia de la limitación acarreada por el principio de redistribución desalud en la proposición de medidas de las desigualdades en salud a partir de la adaptación de las medidas de desigualdad de renta. Los autores apuntan que, a diferencia de la renta, la salud no puede ser redistribuida. Por ejemplo, en lo que concierne a la esperanza de vida, los individuos más favorecidos socialmente no pueden redistribuir años de vida a los menos favorecidos.

En el artículo de revisión de Mackenback \& Kunst (1997), entre los indicadores por ellos discutidos, son citados adicionalmente: (a) razón de superioridad por grupo socioeconómico; (b) índice basado en los efectos de la regresión (lineal, logística, o log-linear), teniendo el NSE como variable independiente; (c) el riesgo atribuible, que indica la reducción relativa en la mortalidad (morbilidad) general, en caso de que toda la población presentase las tasas de mortalidad del grupo de mejor NSE; y (d) el índice de disimilaridad, que mide el apartamiento (diferencias absolutas) entre la distribución dela salud y la distribución de la población por grupo socioeconómico. Los autores enfatizan las diferencias entre los dos últimos: mientras el "índice de disimilaridad" capta las diferencias de la salud por grupo socioeconómico en relación al padrón medio de la población total, el "riesgo atribuible" indica la reducción relativa en la mortalidad (morbilidad) general, en caso de que toda la población presentase las tasas de mortalidad del grupo de mejor NSE.

Manor et al. (1997) analizan el problema de medición de las desigualdades en salud desde el punto de vista estadístico. El problema es examinado a la luz de la hipótesis nula de homogeneidad de la distribución de la enfermedad según los grupos sociales, o de acuerdo con las características de los individuos que componen los agrupamientos. Los autores utilizan índices globales, que miden la distancia entre la distribución esperada bajo la hipótesis de homogeneidad y la distribución observada, así como los efectos parciales, por mediación de la adecuación de regresiones (lineales o logísticas) a los datos, que captan gradientes quizás existentes en las condiciones de salud por subgrupo poblacional.

Murray et al. (1999) proponen que las desigualdades de la salud sean medidas como variaciones entre individuos en la población, sin tener en consideración los determinantes de la salud. Apuntan como ventaja de la perspectiva por ellos sustentada el hecho de que la desigualdad medida en el nivel del individuo, al prescindir de una hipótesis a priori de causalidad, se convertiría, ella misma, en objeto de investigación científica. Como fue apuntado por Braveman et al. (2001), estas medidas reflejan las diferencias entre individuos más saludables 
Medidas de desigualdad en salud más frecuentemente utilizadas en la literatura.

\begin{tabular}{|c|c|c|}
\hline Indicador & Definición & Comentarios \\
\hline Amplitud & $\begin{array}{l}\text { Es calculada como la diferencia de los valores del } \\
\text { indicador de salud encontrados en los dos grupos } \\
\text { socioeconómicos extremos }\end{array}$ & $\begin{array}{l}\text { La medida posee la desventaja de no considerar } \\
\text { los valores del indicador de salud en grupos } \\
\text { socioeconómicos intermedios }\end{array}$ \\
\hline Razón de tasas & $\begin{array}{l}\text { Definida como la razón entre la tasa de morbilidad } \\
\text { (mortalidad) del grupo de mejor NSE y la tasa del } \\
\text { grupo de peor NSE }\end{array}$ & $\begin{array}{l}\text { La medida falla por sólo considerar los polos extremos } \\
\text { de la sociedad }\end{array}$ \\
\hline Coeficiente de Gini & $\begin{array}{l}\text { Este coeficiente está basado en la curva de Lorenz, } \\
\text { construida a partir de las proporciones acumuladas de } \\
\text { la población - ordenada por nivel de salud - y de las } \\
\text { proporciones acumuladas de la medida de salud. } \\
\text { El coeficiente de Gini es estimado por el área entre la } \\
\text { diagonal y la curva de Lorenz, siendo igual a cero, en el } \\
\text { caso de absoluta igualdad, e igual a 1, en el caso de } \\
\text { máxima desigualdad }\end{array}$ & $\begin{array}{l}\text { La medida falla por basarse en el principio de } \\
\text { redistribución de la salud y por no considerar la } \\
\text { dimensión socioeconómica, siendo posible establecer } \\
\text { desigualdades incluso cuando no existe el gradiente del } \\
\text { indicador de salud entre las clases sociales. } \\
\text { Adicionalmente, es intensamente afectada por las } \\
\text { desigualdades socioeconómicas de la población }\end{array}$ \\
\hline Índice de disimilaridad & $\begin{array}{l}\text { Es definido como el porcentaje de casos que deben ser } \\
\text { redistribuidos para que sea posible obtener la misma tasa } \\
\text { de morbilidad o mortalidad para todos los grupos } \\
\text { socioeconómicos }\end{array}$ & $\begin{array}{l}\text { Posee la desventaja de estar fundamentada en el } \\
\text { principio de redistribución de la salud, de los grupos } \\
\text { más favorecidos para los menos favorecidos socialmente. } \\
\text { Falla por evaluar sólo el efecto global, sin distinción de } \\
\text { los efectos parciales de cada grupo social }\end{array}$ \\
\hline $\begin{array}{l}\text { Coeficiente angular } \\
\text { de desigualdad }\end{array}$ & $\begin{array}{l}\text { Es definido como el coeficiente angular de la regresión } \\
\text { lineal entre el indicador de salud y el NSE, teniendo en } \\
\text { consideración el tamaño de cada grupo socioeconómico }\end{array}$ & $\begin{array}{l}\text { Es considerado el mejor indicador cuando las dos } \\
\text { variables - nivel de salud y NSE - pueden ser expresadas } \\
\text { cuantitativamente y una vez que esté sujeto a } \\
\text { modificaciones de acuerdo con el modelo matemático } \\
\text { (por ejemplo, "log-log") que explique más adecuadamente } \\
\text { la relación existente entre las dos variables }\end{array}$ \\
\hline Índice de concentración & $\begin{array}{l}\text { Desarrollado como un perfeccionamiento del coeficiente } \\
\text { de Gini. Incorpora la dimensión socioeconómica en la } \\
\text { construcción de la curva de Lorenz, que, de estar hecha, } \\
\text { está basada en la relación entre las proporciones } \\
\text { acumuladas de la medida de salud frente a las } \\
\text { proporciones acumuladas de la población ordenada } \\
\text { por NSE }\end{array}$ & $\begin{array}{l}\text { Bajo la suposición de un gradiente lineal entre el nivel } \\
\text { de salud y el NSE, el índice de concentración constituye } \\
\text { una medida equivalente al coeficiente angular de } \\
\text { desigualdad. Sin embargo, este índice subestima las } \\
\text { desigualdades en salud en caso de que no exista un } \\
\text { gradiente lineal constante, como en el caso, frecuente, } \\
\text { en que el indicador de salud alcanza un nivel a partir de } \\
\text { un determinado NSE }\end{array}$ \\
\hline $\begin{array}{l}\text { Índice de efecto relativo } \\
\text { basado en regresión }\end{array}$ & $\begin{array}{l}\text { Está fundamentado en la generalización de los } \\
\text { procedimientos epidemiológicos y/o estadísticos } \\
\text { utilizados para comparaciones múltiples del nivel medio } \\
\text { de salud (o de las probabilidades de enfermedad) entre } \\
\text { distintas categorías sociales. Para la construcción de este } \\
\text { índice, se aplica un modelo de regresión (lineal, logística } \\
\text { o logarítmica) teniendo como variable independiente el } \\
\text { NSE, medido como una variable cuantitativa ordinal } \\
\text { o categórica }\end{array}$ & $\begin{array}{l}\text { Dependiendo de las especificaciones del modelo, esta } \\
\text { aproximación produce un índice de efecto relativo } \\
\text { equivalente al tradicional odds-ratio (razón de los } \\
\text { productos cruzados). Utilizando el instrumental } \\
\text { tradicional de la Epidemiología, constituye una medida } \\
\text { bastante adecuada cuando el NSE es expresado por } \\
\text { medio de una variable categórica, permitiendo, } \\
\text { inclusive, comprobar la linealidad del gradiente social }\end{array}$ \\
\hline $\begin{array}{l}\text { Riesgo atribuible en } \\
\text { la población (\%) }\end{array}$ & $\begin{array}{l}\text { Esta medida puede ser interpretada como la presumible } \\
\text { reducción proporcional en la mortalidad general en caso de } \\
\text { que todos los individuos estuviesen expuestos al mismo } \\
\text { riesgo de mortalidad (morbilidad) del grupo de mejor NSE }\end{array}$ & $\begin{array}{l}\text { Se trata de una medida bastante interesante ya que se } \\
\text { refiere a establecer el exceso de óbitos en relación a un } \\
\text { padrón, dado por el grupo de mejor NSE y no por la } \\
\text { media de la población }\end{array}$ \\
\hline
\end{tabular}

NSE = nivel socioeconómico. 
y menos saludables, pero no entre ricos y pobres, entre blancos y negros, o entre hombres y mujeres. La desigualdad más amplia establecida a través de esta estrategia indica sólo la mayor variación en la salud de los individuos de una determinada población, sin que sea posible identificar los factores asociados a estas variaciones.

En el Tabla 1, son presentadas, de forma sinóptica, las medidas de desigualdad en salud más frecuentemente utilizadas en la literatura.

\section{Formulación de un modelo cuantitativo}

Bajo la perspectiva de la inferencia estadística, la hipótesis de igualdad de la situación de salud por nivel socioeconómico es traducida por la hipótesis nula de homogeneidad, para la cual la probabilidad de muerte (enfermedad) es la misma, independientemente de las características socioeconómicas individuales o de los subgrupos poblacionales.

Las medidas globales clásicas - como la $\chi^{2}$ (qui-cuadrado de Pearson) o la L2 (estadística de verosimilitud) -, que miden la distancia de las estimaciones de la probabilidad de incidencia del acontecimiento de salud en los distintos subgrupos poblacionales en relación al padrón esperado por la hipótesis de homogeneidad, no permiten evidenciar, no obstante, en qué consisten estas diferencias. Ya los efectos parciales - traducidos por "coeficientes de regresión", "razón de los productos cruzados" y "riesgo relativo" - se muestran más apropiados a los propósitos de medir las desigualdades en salud, pues consiguen captar gradientes en la salud por subgrupo poblacional.

Mientras tanto, es importante destacar, por encima de todo, que el modelo matemático que fundamenta la relación entre el indicador de salud y el NSE en el nivel individual debe ser tomado en consideración preliminarmente al cálculo de cualquier medida de desigualdad en salud.

En lo tocante a la renta, por ejemplo, se sugiere que el modelo matemático que fundamenta la relación entre el riesgo de mortalidad y la renta, en el nivel individual, sería bien representado por un modelo del tipo "log-log", modelo matemático que consiste en relacionar los logaritmos da tasa de mortalidad a los logaritmos de renta a través de una función lineal (Backlund et al., 1996). De acuerdo con este modelo, para valores de renta $(x)$ menores do que un cierto valor $\left(\mathrm{x}_{0}\right)$, el riesgo de mortalidad $(\mathrm{y})$ decrece con una velocidad no constante, aproximándose de un nivel $\left(\mathrm{y}_{0}\right)$ para valores de renta mayores que el $x_{0}$.
Siendo así, bajo la óptica matemática, una posible aproximación a la cuestión consiste en suponer que el logaritmo del riesgo de mortalidad (y), para un determinado valor de renta (x), pueda ser expresado por:

$\operatorname{Ln}(y)=\operatorname{Ln}\left(y_{0}\right)+\gamma \operatorname{Ln}\left(x / x_{0}\right), x<x_{0}$

$\operatorname{Ln}(y)=\operatorname{Ln}\left(y_{0}\right), x \geq x_{0}$,

donde Ln representa el logaritmo neperiano.

El parámetro $\gamma$ tiene el papel de medir la variación del riesgo de morir por valor de renta: cuanto mayor sea el valor absoluto del parámetro $\gamma$, más pronunciado es el declive del riesgo de mortalidad y mayor la desigualdad del riesgo de morir entre los individuos ricos y pobres. Cuando $\gamma$ es igual a cero, no hay desigualdades, pues el riesgo de morir es constante, y igual a $\mathrm{y}_{0}$, para cualquiera que sea el valor de renta.

La inferencia estadística de que existe igualdad del riesgo de morir, independientemente de la renta del individuo, es, por tanto, equivalente al test de la hipótesis nula $\mathrm{H}_{0}: \gamma=0$. Considerando el modelo log-log, donde $x_{0}$ es un vaIor conocido, el estimador $\hat{\gamma}$ (por mínimos cuadrados o máximo-verosimilitud) constituye una estadística apropiada para la medida de las desigualdades en el riesgo de mortalidad por valor de renta. Ya la estadística padronizada, obtenida por la razón entre $\hat{\gamma}$ y su error padrón, puede ser utilizada para comprobar la hipótesis nula de igualdad.

Se llama la atención sobre el hecho de que la estadística $\hat{\gamma}$ es equivalente al "coeficiente angular de desigualdad", cuando este es estimado por medio de una regresión bajo el presupuesto de que exista una relación "log-log" entre el riesgo de mortalidad y el NSE. Es posible demostrar, entretanto, que si el coeficiente angular de desigualdad fuera calculado mediante la suposición de una relación lineal entre las dos variables, la magnitud de la desigualdad de salud por NSE puede ser bastante afectada, siendo la magnitud de la desigualdad del riesgo de morir entre los individuos pobres y ricos subestimada de forma importante (Szwarcwald \& Bastos, 2001).

Se concluye, así, que examinar la relación entre el indicador de salud bajo análisis y el NSE constituye una etapa fundamental en las aproximaciones metodológicas con vistas a la medición de las desigualdades de salud. Esta etapa debe ser considerada previamente al procedimento de estimación del índice seleccionado para medir las desigualdades en salud. Es también necesario mencionar que el padrón monotónico de disminución no siempre tiene lugar, pudiendo, por tanto, afectar a las medidas de desigualdad en salud que se basan en una relación lineal entre el estado de salud y el NSE. 
Una aplicación: desigualdades de la mortalidad neonatal en Río de J aneiro, 2000

En esta sección son examinados los resultados de un estudio sobre mortalidad infantil desarrollado en el Municipio de Río de Janeiro, en el año de 2000, analizándose las desigualdades del bajo peso al nacer (BPN) y de la mortalidad neonatal según el grado de escolaridad de la madre. Los datos aquí analizados son provenientes del Sistema de Información sobre Mortalidad (SIM) y del Sistema de Nacimientos (SINASC), gentilmente cedidos por la Secretaría Municipal de Salud de Río de Janeiro.

A la luz del ejemplo, son examinados algunos atributos necesarios a la proposición de estadísticas o índices aptos a medir las desigualdades en salud. Un requisito indispensable para una medida de desigualdad socioeconómica en salud es su capacidad de permitir la investigación conjunta del comportamiento de los indicadores de salud frente al comportamiento de los factores socioeconómicos determinantes de las desigualdades, no limitándose, por tanto, a la medida de la dispersión en el ámbito de la distribución del estado de salud en la población. En términos estadísticos, la medida debe partir del análisis de la distribución del estado de salud condicionada a los factores socioeconómicos y no de la distribución marginal del estado de salud de la población.
Eso queda claro cuando se observa la distribución del peso a nacer, referente a Río de Janeiro, para el año de 2000 (Figura 1). El simple análisis de la distribución del peso al nacer, sin que sean investigados los determinantes del bajo peso al nacer (aquí definido como peso menor que $2.500 \mathrm{~g}$ ), no permite evidenciar las desigualdades socioeconómicas relacionadas al BPN. Como ha sido discutido por CarHill (1988), el análisis exclusivo de la variabilidad total del indicador de salud en la población, sin que sean tomadas en cuenta las variaciones sistemáticas provenientes de la adecuada estratificación de la población, no es adecuado.

Ya mediante la apreciación de los resultados dispuestos en la Tabla 2, es posible darse cuenta que la división de la población por grado de escolaridad permite establecer conclusiones sobre el grado de desigualdad de la mortalidad neonatal en Río de Janeiro. Analizando las proporciones de BPN y las tasas de mortalidad neonatal por grado de escolaridad de la madre, se verifican gradientes pronunciados de declive para los dos indicadores de salud por nivel de instrucción de la madre, particularmente, para las tasas de mortal idad neonatal. Estas decrecen de 98, entre las analfabetas, a 9 por 1.000 nacidos vivos (NV), entre aquellas que poseen nivel superior.

Se observa, igualmente, que la velocidad de declive no es constante, siendo el declive bastante más pronunciado entre la categoría de

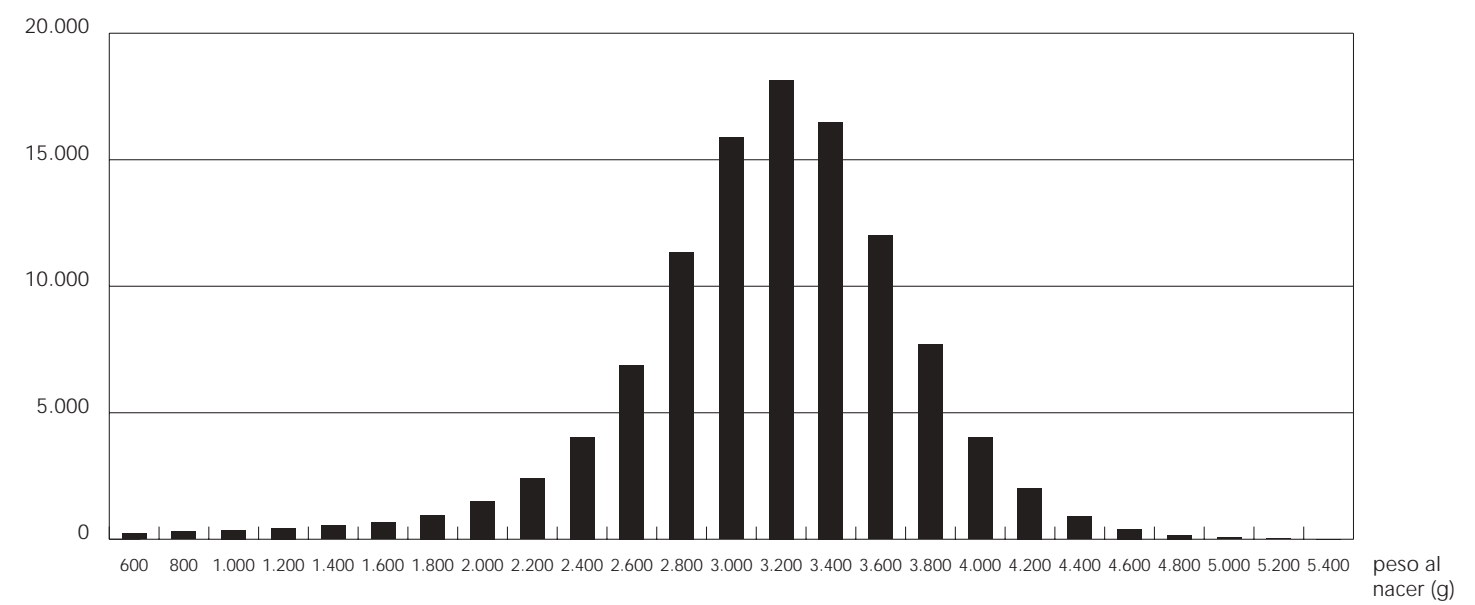


Tabla 2

Tasa de mortalidad neonatal (/1.000 nacidos vivos) y proporción (\%) de nacidos vivos con bajo peso al nacer $(<2.500 \mathrm{~g})$ por grado de escolaridad de la madre. Municipio de Río de J aneiro, 2000.

\begin{tabular}{lcc}
\hline Escolaridad de la madre & Mortalidad neonatal & Proporción de BPN (\%) \\
\hline Analfabeta & 98,1 & 13,9 \\
Hasta el 1o grado & 12,8 & 11,1 \\
2o grado & 8,5 & 10,3 \\
Superior & 8,7 & 9,1 \\
Total & 15,0 & 10,4 \\
\hline
\end{tabular}

BPN = bajo peso al nacer. ción de un único valor, igual a 15,0 por 1.000 $\mathrm{NV}$, para el riesgo de morir en el período neonatal, independientemente del grado de escolaridad de la madre. Cabe observar que el valor único de la tasa de mortalidad neonatal corresponde al valor encontrado para el total de la población. Como la igualdad es alcanzada cuando el índice de disimilaridad es igual a cero, la igualdad, de acuerdo con esta medida, sería alcanzada por la redistribución de las muertes en el período neonatal entre las categorías de escolaridad de la madre.

Una medida más adecuada puede ser obtenida mediante el cálculo del riesgo atribuible, según el cual el exceso de muertes en cada categoría de escolaridad es estimado con base en la tasa de mortalidad neonatal mínima alcanzada en el grupo de madres con nivel de instrucción 2o grado/superior. Se considera, todavía, que más importante que resumir las desigualdades socioeconómicas de la salud en un único índice es proporcionar evidencias que ellas existen, y describir la manera como ellas se producen en el espectro de las variaciones socioeconómicas.

Manor et al. (1997), al discutiren las ventajas y desventajas de diferentes medidas de desigualdades en salud, encontraron gran variación en la magnitud de las desigualdades de acuerdo con el indicador utilizado para medir la posición social de los individuos. Una de las formas más frecuentemente utilizadas para caracterizar el NSE es la medida de la renta (Chandola, 2000; Winkleby et al., 1992), que puede ser analizada tanto en el nivel individual como colectivamente, através de la renta familiar (Arber, 1991). Cabe observar que, mientras la renta representa una situación actual, la escolaridad es una variable más estable en el sentido de representar un bagaje adquirido en el pasado, que no se pierde con el tiempo. La escolaridad no depende, por ejemplo, de la inserción actual del individuo en el mercado de trabajo o de la naturaleza de la ocupación que él desempeña en un dado momento (Feldman et al., 1989; Sorlie et al., 1995). Teniendo en vista que el entendimiento de las desigualdades en salud encuentra explicaciones en la experiencia acumulada del individuo en diferentes fases de vida (Lynch et al., 1994; Power \& M atthews, 1997), el grado de educación es particularmente importante. Sin embargo, la escolaridad presenta la desventaja de tener pequeña capacidad discriminante para ciertos eventos de salud, principalmente en los países desarrollados, donde la mayoría de la población tiene por lo menos la enseñanza fundamental.

La ocupación también ha sido utilizada como una proxy del NSE. Las ocupaciones son 
generalmente agrupadas por rama de actividad o posición relativa en el conjunto de ocupaciones, expresando, por un lado, el grado de escolaridad compatible con el nivel de conocimiento exigido para el ejercicio de la función, y, por otro, la renta proveniente de la remuneración atribuida al individuo que ejerce aquella función específica en un dato contexto socioeconómico (Diderichsen \& Hallqvist, 1997; Mach \& Wesolowski, 1986). Las dificultades mayores de la utilización de la "ocupación" se refieren a la eventual clasificación de los individuos no incluidos en la población económicamente activa (PEA), como los jubilados y las mujeres involucradas en actividades domésticas (Arber, 1996).

En el caso del SIM, sólo es posible obtener informaciones sobre ocupación y grado de escolaridad de la madre. Limitaciones adicionales como el uso de la ocupación como variable indicadora del NSE son derivadas del expresivo porcentaje de mujeres clasificadas como domésticas, sin mayores especificaciones que permitan distinguir las "amas de casa", que, generalmente, pertenecen a los grupos de mayor renta, de las "empleadas domésticas", que, en contraste, forman parte de los grupos más desfavorecidos en la escala social.

Aunque las limitaciones de los datos disponibles en el ejemplo aquí analizado no hayan permitido el análisis por ocupación, restringiéndose, por tanto, el estudio a los análisis por grado de escolaridad, la apreciación de las informaciones por nivel de instrucción materno permite inferir que el modelo que relaciona la mortalidad neonatal al NSE de la madre no es lineal, observándose un amplio gradiente entre las madres analfabetas y las madres con instrucción fundamental, que disminuye progresivamente, a medida que aumenta el grado de instrucción.

En este sentido, precauciones adicionales deben ser tenidas en cuenta como, por ejemplo, la de la estimación de medidas como el "coeficiente angular de desigualdades". El gráfico, presentado en la Figura 2, basado en asociar los valores de la tasa de mortalidad infantil a los valores de renta media en los barrios de Río de Janeiro, muestra que la suposición de un modelo lineal para fundamentar la relación entre el riesgo de morir y la renta afecta, considerablemente, al coeficiente angular de desigualdades. Resáltese, sobre todo, que al no tenerse en cuenta más tarde la existencia de una categoría determinada de nivel de renta, se puede incurrir en subestimaciones importantes de las desigualdades socioeconómicas de la salud.

Otro punto fundamental en el ámbito de la problemática de la medida de las desigualda-
Figura 2

Variación de la tasa de mortalidad infantil por renta.

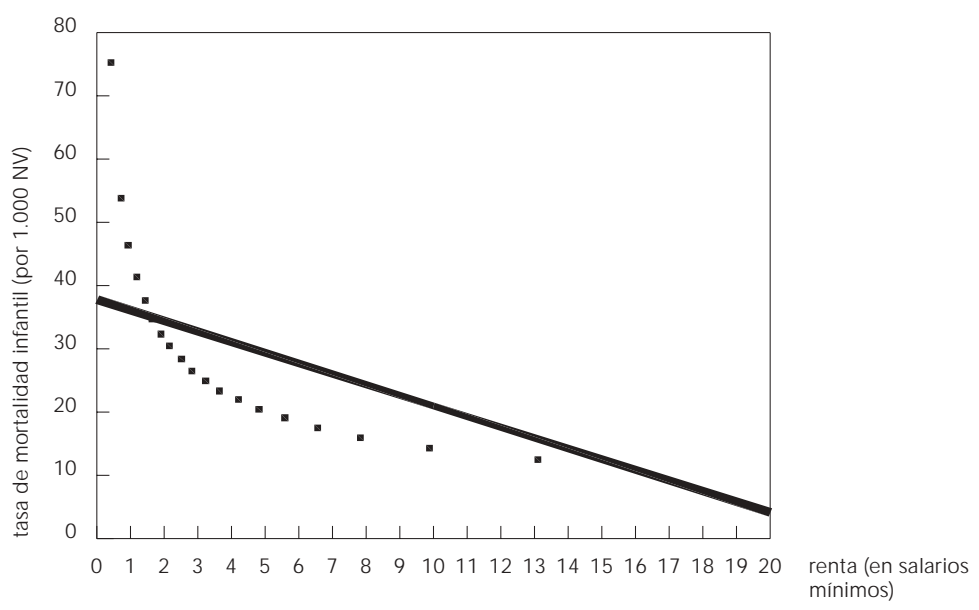

des en salud fue formulado por la OMS, al proponer que las desigualdades en salud constituyesen una de las dimensiones para la evaluación del desempeño de los sistemas de salud (WHO, 2000). Bajo esta perspectiva, la actuación de los sistemas de salud constituye un elemento fundamental de una posible (y deseada) disminución de las desigualdades.

Los datos dispuestos en la Tabla 3 permiten verificar el efecto relevante de la atención prenatal. Las desigualdades del BPN por grado de escolaridad de la madre disminuyen cuando se considera el efecto protector proporcionado por una atención pre-natal adecuada.

Por tanto, en la evaluación de las acciones de salud que objetivan la reducción de las desigualdades, la consideración exclusiva de los determinantes socioeconómicos no es suficiente. El desafío está, justamente, en construir una red de causalidad capaz de representar no sólo las relaciones entre los factores socioeconómicos y el indicador de salud bajo análisis, sino también los efectos que intervienen en las acciones de salud, que, conjuntamente con los primeros, pueden afectar intensamente la respuesta.

\section{Comentarios finales}

La cuestión de las relaciones entre las desigualdades sociales y económicas y las condiciones de salud de las poblaciones están siendo vigorosamente retomadas por la investigación con- 
Tabla 3

Proporción (\%) de recién-nacidos con bajo peso al nacer según el número de consultas de atención pre-natal y el grado de escolaridad de la madre. Municipio de Río de J aneiro, 2000.

\begin{tabular}{llr}
\hline Escolaridad de la madre & Atención pre-natal & $\%$ \\
\hline Analfabeta & $0-3$ consultas & 18,0 \\
& 4 y más consultas & 12,0 \\
Hasta el lo grado & 0 -3 consultas & 18,6 \\
& 4 y más consultas & 9,3 \\
2o grado & $0-3$ consultas & 22,0 \\
& 4 y más consultas & 9,2 \\
Superior & 0 -3 consultas & 19,4 \\
& 4 y más consultas & 8,4 \\
\hline
\end{tabular}

temporánea (Birch, 2001; Blane, 2001) y se reviste de gran relevancia y premura por dos razones fundamentales. Por un lado, porque aumentan las evidencias que, consistentemente, indican que los factores sociales constituyen elementos centrales en la determinación de los padrones de morbi-mortalidad de las sociedades. Por otro, porque de manera consensual, se constata que la desigualdad en el ámbito de la salud debe ser enfrentada no sólo a través del compromiso ético con la transformación político-social y la mayor equidad económica (Charlton, 1994). Es preciso definir claramente estrategias de reducción de las desigualdades en salud, a través de alternativas objetivas, pasibles de medida y evaluación, pragmáticas y viables, aunque en circunstancias de restricción presupuestaria.

Evidencias recientes no sólo de persistencia, pero también de aumento de la desigualdad de la salud, en varios países, a lo largo del tiempo, han Ilamado aún más la atención sobre el tema (Diderichsen \& Hallqvist, 1997; Marang-van de $M$ heen et al., 1998; Pappas et al., 1993). La hipótesis explicativa más frecuente para el aumento de la disparidad contempla el mayor declive de la mortalidad entre los grupos de mayor nivel socioeconómico (Turrell \& Mathers, 2001). Explicación complementaria es sugerida por Victora et al. (2000) al analizaren los efectos de intervenciones específicamente dirigidas a la salud materno-infantil en Brasil. Los autores sugieren que nuevas intervenciones afectarían, primeramente, aquellos con mejor condición social, lo que resultaría, al principio, en un aumento de la desigualdad.
La reducción de la desigualdad aparecería con el tiempo, cuando los pobres obtuviesen amplio acceso a las intervenciones y los ricos alcanzasen el nivel de mortalidad (morbilidad), además de que eso sería improbable para conseguir mejoras substanciales.

El presente trabajo contempla un breve análisis acerca de las medidas de desigualdad en salud, con una ampliación práctica de los procedimientos metodológicos y conceptos anteriormente discutidos a la luz de la medición de las desigualdades de la mortalidad infantil precoz, cuestión central de la salud pública en el contexto brasileño.

Analizada según el grado de escolaridad de la madre, la mortalidad neonatal en la ciudad de Río de Janeiro demostró disparidades relevantes, sobre todo en el grupo compuesto por las madres analfabetas en relación a las demás. La actuación del sistema de salud, traducida por la atención pre-natal, mostró un efecto importante, que podría ser todavía más efectivo en la reducción de las desigualdades de la mortalidad infantil mediante políticas dirigi das a la cobertura que más abarca a la población-objetivo. Resultados semejantes fueron discutidos por Whitehead \& Dahlgen (1991), que destacan que una reducción de un $20 \%$ en la mortalidad perinatal, en Gran Bretaña, podría ser obtenida por la implementación universal de la atención pre-natal de calidad adecuada.

A pesar de las disparidades presentes, históricamente, en nuestra sociedad, y que se manifiestan de las formas más diferentes, la investigación de las desigualdades en salud en la población brasileña no ha merecido la atención necesaria. Iniciativa reciente, desarrollada en conjunto por el Centro Nacional de Epidemiología del Ministerio de la Salud (MS), Instituto de Investigación Económica Aplicada y Organización Pan-Americana de la Salud (Viana et al., 2001), evidenció importantes desigualdades en los indicadores de salud estudiados, que incluyeron no sólo indicadores de condiciones de salud, sino también indicadores de oferta, acceso y utilización de servicios de salud. Aunque los resultados hayan, de alguna forma, enfatizando las desigualdades de salud, el análisis de las variaciones tuvo que restringirse a $\mathrm{ni}$ vel ecológico, sobre todo por la indisponibilidad de informaciones específicas sobre el nivel socioeconómico de los individuos a nivel nacional.

Es probable que la escasez de estudios nacionales sobre el tema sea parcialmente explicada por la limitación de los datos disponibles en el nivel nacional. Esfuerzos dirigidos al perfeccionamiento de los sistemas de información 
del MS, como el SIM, el SINASC y el Sistema de Informação Hospitalar do Sistema Único de Saúde (SIH/SUS), y encaminados a la implementación de investigaciones por muestras con periodicidad regular en el sentido de obtención de datos específicos sobre la condición social de los individuos son, de esta forma, imprescindibles para el análisis de las desigualdades socioeconómicas de la salud en el país (Travassos et al., 2000).

La monitoreo de las desigualdades en salud, en diferentes dimensiones que no es exclusivamente la geográfica, se configura como esencial para el proceso de evaluación del sistema de salud brasileño. No sólo porque la equidad constituye uno de los principios que guían el SUS, sino también porque se cree que es posible reducir las desigualdades en salud mediante acciones efectivas, aunque en un contexto de enorme y reconocida disparidad en la distribución de renta. Teniendo en cuenta que no es posible redistribuir la salud, es necesario definir lo más explícitamente posible cómo es, y, en qué medida las desigual dades socioeconómicas se interrelacionan con la inequidad en salud, identificando segmentos más vulnerables al impacto de políticas compensatorias y subsidiando acciones que encontrarán mayor eco en los grupos y comunidades más desfavorecidas socialmente.

\section{Referencias}

ACHESON, D., 2000. Health inequalities impact assessment. Bulletin of theWorld Health Organization, 78:75-85.

ADLER, N.; BOYCE, W. T.; CHESNEY, M. A.; FOLKMAN, S. \& SYME, S. L., 1993. Socioeconomic differences in health: No easy solution. JAMA, 269: 3140-3145.

ARBER, S., 1991. Class, paid employment and family roles: Making sense of structural disadvantage, gender and health status. Social Scienceand Medicine, 32:425-436.

ARBER, S., 1996. Integrating nonemployment into research on health inequalities. International Journal of Health Services, 26:445-481.

BACKLUND, E.; SORLIE, P. D. \& JOHNSON, N. J., 1996. The shape of the relationship between income and mortality in the United States. Annals of Epidemiology, 6:12-20.

BERKM AN, L. F. \& KAWACHI, I. (ed.), 2000. Social Epidemiology. Oxford: Oxford University Press.

$\mathrm{BIRCH}, \mathrm{S} ., 2001$. Commentary: Social inequalities in health, social epidemiology and social value. International Journal of Epidemiology, 30:294-296.

BLANE, D., 2001. Commentary: Socioeconomic health differentials. International Journal of Epidemiology, 30:292-293.
BRAVEMAN, P.; STARFIELD, B. \& GEIGER, H. J., 2001. World health report 2000: How it removes equity from the agenda for public health monitoring and policy. BMJ, 323:678-681.

CARR-HILL, R., 1988. Time trends in inequalities in health. Journal of Biosocial Science, 20:265-273.

CHANDOLA, T., 2000. Social class differences in mortality using the new UK National Statistics SocioEconomic Classification. Social Science and Medicine, 50:641-649.

CHARLTON, B. G., 1994. Is inequality bad for national health? Lancet, 343:221-222.

DIDERICHSEN, F. \& HALLQVIST, J., 1997. Trends in occupational mortality among middle aged men in Sweden 1961-1990. International Journal of Epidemiology, 26:782-787.

DUNCAN, G. J., 1996. Income dynamics and health. International Journal of Health Services, 26:419-444.

EVANS, R. G.; BARER, M. L. \& MARM OR, T. R., (ed.), 1994. Why are Some People Healthy and Others Not? The Determinants of Health of Populations. New York: Aldine de Gruyter.

FELDMAN, J. J.; MAKUC, D. M.; KLEINMAN, J. C. \& CORNONI-HUNTLEY, J., 1989. National trends in educational differentials in mortality. American Journal of Epidemiology, 129:919-933. 
GAKIDOU, E. E.; MURRAY, C. J. L. \& FRENK, J., 2000. Defining and measuring health inequality: An approach based on the distribution of health expectancy. Bulletin of theWorld Health Organization, 78:42-54.

GWATKIN, D. R., 2000. Health inequalities and the health of the poor: What do we know? What can we do? Bulletin of theWorld Health Organization,78:3-18.

HOWELLING, T. A. J.; KUNST, A. E. \& MACKENBACH, J. P., 2001. World health report 2000: Inequality index and socioeconomic inequalities in mortality. Lancet, 357:1671-1672.

KAPLAN, G. A., 1996. People and places: Contrasting perspectives on the association between social class and health. International Journal of Health Services, 26:507-519.

KAPLAN, G. A. \& KEIL, J. E., 1993. Socioeconomic factors and cardiovascular disease: A review of the literature. Circulation, 88:1973-1998.

KNUST, A. E.; GEURTS, J. J. M. \& van den BERG, J., 1995. International variation in socioeconomic inequalities in self reported health. Journal of Epidemiology and Community Health, 49:117-123.

LYNCH, J. H.; KAPLAN, G. A.; COHEN, R. D.; KAUHANEN, J.; WILSON, T. W.; SMITH, N. L. \& SALONEN, J. T., 1994. Childhood and adult socioeconomic status as predictors of mortality in Finland. Lancet, 343:524-527.

MACH, B. W. \& WESOLOWSKI, W., 1986. Social Morbidity and Social Structure. London: Routledge \& Kegan Paul.

MACKENBACH, J. P. \& KUNST, A. E., 1997. Measuring the magnitude of socio-economic inequalities in health: An overview of available measures illustrated with two examples from Europe. Social Science and Medicine, 44:757-771.

MACKENBACH, J. P.; KUNST, A. E.; CAVELAARS, A. E. J. M.; GRONHOF, F.; GEURTS, J. J. M. \& THE EU WORKING GROUP ON SOCIOECONOMIC INEQUALITIES IN HEALTH, 1997. Socioeconomic inequalities in morbidity and mortality in western Europe. Lancet, 349:1655-1659.

MANOR, O.; MATTHEWS, S. \& POWER, C., 1997. Comparing measures of health inequality. Social Science and Medicine, 45:761-771.

MARANG-van de MHEEN, P. J.; DAVEY-SMITH, G.; HART, C. L. \& GUNNING-SCHEPERS, L. J., 1998. Socioeconomic differentials among men within Great Britain: Time trends and contributory causes. Journal of Epidemiology and Community Health, 52:214-218.

MURRAY, C. J. L.; GAKIDOU, E. E. \& FRENK, J., 1999. Health inequalities and social group differences: What should we measure? Bulletin of theWorld Health Organization, 77:537-543.

PAMUK, E. R., 1985. Social class and inequality in mortality from 1921 to 1972 in England and Wales. Population Studies, 39:17-31.

PAPPAS, G.; QUEEN, S.; HADDEN, W. \& FISHER, G., 1993. The increasing disparity in mortality between socioeconomic groups in the United States 1960 and 1986. New England Journal of Medicine, 329:103-109.

POWER, C. \& MATTHEWS, S., 1997. Origins of health inequalities in a national population sample. Lancet, 350: 1584-1589.
SANTOS, M., 1994. Por uma Economia Política da Cidade. São Paulo: Editora Hucitec.

SORLIE, P. D.; BACKLUND, E. \& KELLER, J. B., 1995. US mortality by economic, demographic, and social characteristics: The National Longitudinal Mortality Study. American Journal of Public Health, 85:949-956.

SZWARCWALD, C. L., 2002. On the WHO's measurement of health inequalities. Journal of Epidemiology and Community Health, 56:177-182.

SZWARCWALD, C. L. \& BASTOS, F. I., 2001. On the measurement of health inequalities by incomes classes. In: Nordic Conference in Social Medicine $\&$ Public Health, Proceedings, October 2001 বttp: // www.uib.no/isf/social/conf2001>.

TOWNSEND, P. \& DAVIDSON, N., 1982. Inequalities in Health: The Black Report and The Health $\mathrm{Di}$ vide. Harmondworth: Penguin.

TRAVASSOS, C.; SANTOS, S. M.; SZWARCWALD, C. L.; BARCELLOS, C.; ROMERO, D. E.; BASTOS, F. I.; VIACAVA, F. \& AZEVEDO, L. O., 2000. Variáveis sociais nos bancos de dados de interesse para a área da saúde. In: I Seminário Nacional de Informação e Saúde. Anais, pp. 35-43, Rio de Janeiro: Editora Fiocruz.

TURRELL, G. \& MATHERS, C., 2001. Socioeconomic inequalities in all-cause and specific-cause mortality in Australia: 1985-1987 and 1995-1997. International Journal of Epidemiology, 30:231-239.

VIANNA, S. M.; NUNES, A.; SANTOS, J. R. S. \& BARATA, R. B., 2001. Medindo as Desigualdades em Saúde no Brasil. Brasília: Organização Pan-Americana da Saúde/ Organização Mundial da Saúde.

VICTORA, C. G.; VAUGHAN, J. P.; BARROS, F. C.; SILVA, A. C. \& TOMASI, E., 2000. Explaining trends in inequities: Evidence from Brazilian child health studies. Lancet, 356-1093-1098.

WAGSTAFF, A., 2000. Socioeconomic inequalities in child mortality: Comparisons across nine developing countries. Bulletin of theWorld Health Organization, 78:19-29.

WAGSTAFF, A.; PACI, P. \& van DOORSLAER, E., 1991. On the measurement of inequalities in health. Social Science and Medicine, 33:545-557.

WHITEHEAD, M., 1992. The concepts and principles of equity and health. International Journal of Health Services, 22:429-445.

WHITEHEAD, M. \& DAHLGREN, G., 1991. What can be done about inequalities in health? Lancet, 338:1059-1063.

WINKLEBY, M. A.; JATULIS, D. E.; FRANAK, E. \& FORTMANN, S. P., 1992. Socioeconomic status and health: How education, income, and occupation contribute to risk factors for cardiovascular disease. American Journal of Public Health, 82: 816-20.

WOODWARD, A. \& KAWACHI, I., 2000. Why reduce health inequalities? Journal of Epidemiology and Community Health, 54:923-929.

WHO (World Health Organization), 2000. TheWorld Health Report 2000. Geneva: WHO.

Recibido el 5 de diciembre de 2001

Versión final presentada el 21 de enero de 2002

Aprobado el 9 de abril de 2002 